\title{
Nivel de consumo de alcohol y rendimiento académico en estudiantes universitarios del estado de Hidalgo, México
}

\author{
Alcohol consumption level and academic performance in college students from the state of \\ Hidalgo, Mexico

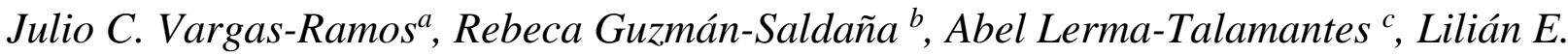 Bosques-Brugada ${ }^{d}$, Angélica Romero-Palencia ${ }^{e}$

\begin{abstract}
:
College students can interact with risk factors that are related with high levels of alcohol consumption, which usually affect academic performance. The aim of this study is to describe the alcohol consumption level and his relation with academic performance on a college student's sample. With a not experimental and correlational design, the AUDIT questionnaire and a sociodemographic data survey (purposefully designed) were applied in a college students sample $(\mathrm{N}=341)$ with an age range of 18 to 25 years old $\left(\mathrm{X}_{\text {age }}=\right.$ $19.8, \mathrm{SD}=2.13$ ) belonging to the degree in Psychology, in the state of Hidalgo, Mexico. On the results, we observed that $76 \%$ of the sample consumes alcohol, and most of the students present a low risk consumption level (57.8\%), 14.1\% of the students present high risk consumption level, and $4.1 \%$ present dependence risk consumption level; finally, alcohol consumption has a low and significant relation with grade point average $(R=-.232, p<.001)$ and failed subjects $(R=.194, p<.001)$. In conclusion, the descriptive results that were obtained in this study are similar to those described in national reports; also, the alcohol consumption has a significant relation with the academic performance, which evidences the need to carry out actions that prevent and reduce this problem.
\end{abstract}

Keywords:

Alcohol consumption, academic performance, college students

\section{Resumen:}

Los estudiantes universitarios pueden interactuar con factores de riesgo relacionados con índices elevados de consumo de alcohol, que suelen afectar el rendimiento académico. El presente estudio tiene como objetivo describir el nivel de consumo de alcohol y su asociación con el rendimiento académico en estudiantes universitarios. Utilizando un diseño no experimental, con un tipo de estudio correlacional, se aplicó el Cuestionario AUDIT; así como una encuesta (diseñada ex profeso) de datos sociodemográficos, en una muestra $(\mathrm{N}=341)$ de estudiantes universitarios con un rango de edad de 18 a 25 años $\left(\mathrm{X}_{\text {edad }}=19.8, \mathrm{DE}=2.13\right)$, pertenecientes a la licenciatura en Psicología (en el estado de Hidalgo, México). En los resultados se observa que el 76\% de la muestra consume alcohol; la mayoría de los participantes presentan un nivel de consumo "sin riesgo" (57.8\%), mientras que el $14.1 \%$ presenta un "consumo de riesgo" y $4.1 \%$ tiene un consumo "con riesgo de dependencia"; por otro lado, el consumo de alcohol presenta una relación débil pero significativa con el promedio académico $(R=-.232, p<.001)$ y el número de materias reprobadas $(R=.194, p<.001)$. En conclusión, los resultados descriptivos obtenidos son similares a los reportados a nivel nacional; también el consumo de alcohol está relacionado de manera significativa con el rendimiento académico, lo cual evidencia la necesidad de realizar acciones que prevengan y disminuyan esta problemática.

\section{Palabras Clave:}

a Autor de Correspondencia, Universidad Autónoma del Estado de Hidalgo, https://orcid.org/ 0000-0002-6803-5686, Email: ju1940810@gmail.com

b Universidad Autónoma del Estado de Hidalgo, https://orcid.org/0000-0003-0877-4871, Email: rguzman@uaeh.edu.mx c Universidad Autónoma del Estado de Hidalgo, https://orcid.org/0000-0001-7212-641X, Email: abel_lerma@uaeh.edu.mx d Universidad Autónoma del Estado de Hidalgo, https://orcid.org/0000-0002-3969-683X, Email: lilian_bosques@uaeh.edu.mx e Universidad Autónoma del Estado de Hidalgo, https://orcid.org/0000-0003-0933-6937, Email: angelica_romero@uaeh.edu.mx 


\section{Introducción}

El consumo excesivo de alcohol continúa siendo una problemática de salud que afecta de manera importante a todo el mundo. ${ }^{1-3}$ En México, de acuerdo a la Encuesta Nacional de Consumo de Drogas, Alcohol y Tabaco ENCODAT, 2016-2017, ${ }^{4} 51.9 \%$ de los hombres y $24 \%$ de las mujeres adultos consumen alcohol de manera excesiva, siendo los estados de Jalisco, Aguascalientes y Colima, las entidades en donde hay una mayor prevalencia de bebidas embriagantes; también se reporta que $53.1 \%$ de la población inició su consumo a los 17 años o menos, y que $4.6 \%$ de los hombres y $0.6 \%$ de las mujeres presentan dependencia al consumo de alcohol.

En cuanto al estado de Hidalgo, se reportan prevalencias de consumo similares al promedio nacional; sin embargo, en los rubros de "consumo excesivo en el último mes" y "dependencia al alcohol", se reportan prevalencias menores. ${ }^{4}$ De acuerdo con la Encuesta Nacional de Salud y Nutrición ENSANUT 2018. 5, 25.3\% de la población de Hidalgo, que tienen una edad entre los 10 y los 19 años, han consumido alcohol al menos una vez en su vida; en cuanto a la población mayor a 20 años, se reporta que $14.5 \%$ afirma consumir alcohol de manera semanal o diariamente.

\section{Consumo en estudiantes universitarios}

Los estudiantes universitarios son un tipo de población que presenta niveles elevados de consumo de alcohol, incluso al compararse con la población de adultos en general. ${ }^{6,7}$ Se han relacionado diversos factores de riesgo con esta problemática, los cuales pueden agruparse en tres categorías: a) factores macrosociales, b) factores microsociales, y c) factores personales. ${ }^{8}$ Los primeros involucran el entorno en el que se encuentra el estudiante, por ejemplo, la disponibilidad del alcohol, el escaso control de su venta, la aprobación por parte de la población de esta conducta, y la publicidad en los medios de comunicación relacionada al consumo de bebidas alcohólicas; los segundos hacen referencia al círculo social del estudiante, como antecedentes de consumo excesivo en la familia cercana, falta de comunicación con padres o tutores, un estilo de crianza permisivo o sobreprotector, y un círculo de amigos o pareja que consumen alcohol $\mathrm{u}$ otras sustancias; finalmente, los factores personales son aquellas características y rasgos propios del estudiante, como desinformación acerca del alcohol, actitudes y creencias favorables hacia el consumo, estados de ansiedad y estrés elevado, y problemas personales. ${ }^{9-13}$

A estos factores también se les suma que regularmente los estudiantes universitarios cuentan con la mayoría de edad por lo que tienen una mayor facilidad de adquirir bebidas alcohólicas; que se encuentran en la última etapa de la adolescencia, la cual se caracteriza por cambios biológicos, psicológicos y sociales, y la búsqueda de experiencias novedosas e independencia social; también hay una disminución en cuanto a la supervisión por parte de los padres o tutores, debido principalmente a que varios estudiantes tienen la necesidad de cambiar de residencia; finalmente, la carga de trabajo escolar propia de la educación profesional es otro factor a considerar que puede relacionarse al consumo excesivo de alcohol. ${ }^{11,14-}$ 16

Por otro lado, un consumo elevado de alcohol recurrente puede generar consecuencias negativas para el estudiante universitario como deserción escolar y una disminución en su rendimiento académico expresado en un bajo promedio de calificaciones y un alto índice de reprobación; $;, 7$ también la realización de diversas conductas de riesgo al estar bajo los efectos del alcohol, como tener relaciones sexuales sin protección, consumir sustancias ilegales, y ejercer violencia contra otras personas. ${ }^{14}$ Aunado a esto, de acuerdo con un estudio realizado por Guerrero-López y colaboradores. 17 en México, se estima que entre los años 2000 y 2011, 20.4\% de las muertes por accidente vial en adolescentes, estuvieron relacionadas con el consumo de alcohol.

Debido a las consecuencias negativas que pueden presentar los estudiantes universitarios que consumen alcohol de manera recurrente, surge la necesidad de conocer los niveles de consumo presentes en este tipo de población, así como la relación de este último con el rendimiento académico de los estudiantes, y así poder realizar las acciones necesarias para prevenir y disminuir esta problemática. ${ }^{18-20}$ Por lo tanto, este estudio tiene como objetivos, describir el nivel de consumo de alcohol, y establecer la asociación entre este y el rendimiento académico en una muestra de estudiantes universitarios de la carrera de Psicología, en el estado de Hidalgo, México, a través de un diseño no experimental y de tipo correlacional.

\section{Método}

\section{Participantes}

Se consideró una muestra de estudiantes universitarios pertenecientes a la licenciatura de Psicología, la cual 
estuvo conformada por $\mathrm{N}=341$ participantes ( 250 mujeres [73.3\%] y 91 hombres [26.7\%]) con un rango de edad entre 18 y 25 años y una edad promedio de 19.8 años (DE $=2.13)$. Del total de la muestra, 330 participantes $(96.8 \%)$ son solteros, 7 participantes (2.1\%) están en unión libre y 4 participantes (1.2\%) son casados. El muestreo utilizado fue no probabilístico, por conveniencia.

\section{Instrumentos de Evaluación}

Se les brindó a los estudiantes una encuesta (diseñada ex profeso) para obtener datos sociodemográficos, como edad, sexo, semestre en el que está cursando, si vive sólo o acompañado y si es un estudiante local o foráneo. El rendimiento académico, evaluado a través del promedio y el número de materias reprobadas de los participantes, fue brindado por las autoridades administrativas de la licenciatura donde fue llevado a cabo el estudio.

Para evaluar el nivel de consumo de alcohol en los participantes, se utilizó el Cuestionario de Identificación de los Trastornos debidos al Consumo de Alcohol (AUDIT) ${ }^{21}$, el cual consta de 10 reactivos con cinco opciones de respuesta tipo Likert y tienen un valor asignado entre el 0 al 4 . Un puntaje igual a 0 indica un "consumo nulo", un puntaje entre 1 y 7 indica un "consumo sin riesgo", un puntaje entre 8 y 15 indica un "consumo de riesgo" y un puntaje igual o mayor a 16 indica un consumo "con riesgo de dependencia". El Cuestionario fue validado para población mexicana, obteniendo altos índices de confiabilidad y validez. ${ }^{22}$

\section{Procedimiento}

Posterior a que el protocolo de la investigación fuera aprobado por el Comité de Ética e Investigación de la Universidad en la que se aplicó este estudio, se solicitó la autorización a las autoridades competentes de la licenciatura en Psicología para realizar la aplicación de los instrumentos en la población estudiantil. Se procedió a invitar a los estudiantes a participar, se les brindó un consentimiento informado y la aplicación fue realizada en las aulas de clases, apoyados por tres licenciados en psicología previamente capacitados.

\section{Análisis Estadístico}

Se utilizó estadística descriptiva para obtener medidas de tendencia central y dispersión del nivel de consumo de alcohol de la muestra, así como otro tipo de variables obtenidas en la encuesta de datos sociodemográficos; también se realizó una correlación de Pearson para establecer la asociación entre el consumo de alcohol y el rendimiento académico; para esto, se utilizó el software IBM-SPSS para Windows, en su versión 21 en español.

\section{Resultados}

En la Tabla 1 se presentan los resultados obtenidos de la aplicación del AUDIT y de la encuesta de datos sociodemográficos aplicada en la muestra.

Tabla 1. Resultados de la evaluación del consumo de alcohol

\begin{tabular}{lc}
\hline Estudiantes universitarios $(\mathbf{n}=\mathbf{3 4 1})$ & Frecuencia \\
\hline $\begin{array}{l}\text { Días por semana que consume } \\
\text { alcohol }\end{array}$ & $1.27 \pm 1.27^{\star}$ \\
Puntaje (AUDIT) & $4.23 \pm 4.84^{\star}$ \\
Nivel de consumo (AUDIT) & \\
Nulo & 82 \\
Sin riesgo & 197 \\
Con riesgo & 48 \\
Con riesgo de dependencia & 14 \\
Consume alcohol & \\
Días Lunes, Martes y Miércoles & 38 \\
Días Jueves y Viernes & 176 \\
Días Sábado y Domingo & 125 \\
Al inicio del semestre & 118 \\
A mediados del semestre & 101 \\
Al final del semestre & 200 \\
\hline
\end{tabular}

${ }^{*}$ Puntaje correspondiente a media y desviación estándar

En los resultados obtenidos, cabe destacar que 197 participantes (57.8\%) obtuvieron un puntaje en el AUDIT correspondiente a "consumo sin riesgo", siendo este nivel de consumo el más frecuente en la muestra, seguido por el "consumo nulo" el cual estuvo presente en 82 participantes $(24 \%)$, y finalmente un total de 48 participantes $(14.1 \%)$ presentaron "consumo de riesgo".

También se destaca que 176 estudiantes (51.6\%) refiere consumir alcohol los días jueves y viernes, siendo los más comunes en la muestra; y el periodo correspondiente al final del semestre es en donde más alumnos (58.7\%) describen que es más probable que consuman alcohol.

Por otro lado, en la Figura 1 se describe el tipo de consumo de alcohol de la muestra de acuerdo al AUDIT, haciendo una comparación por sexo de los estudiantes. En esta, se observa que en el nivel de "consumo nulo" y "consumo sin riesgo", las mujeres (26.8\%, 59.6\%) presentan una prevalencia ligeramente mayor en comparación con los hombres (16.5\%, 52.7\%), mientras que en los niveles de "consumo con riesgo" y "consumo con riesgo de dependencia" los hombres (20.9\%, 9.9\%) presentan una mayor prevalencia en comparación con las mujeres $(11.6 \%, 2 \%)$. 


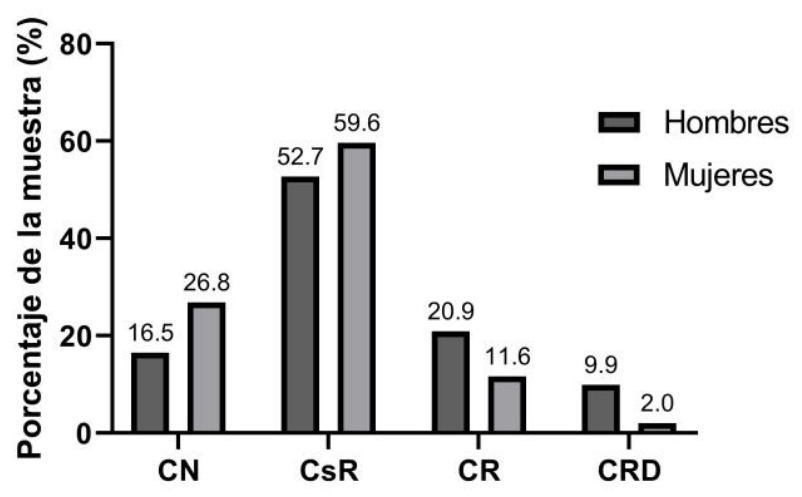

Figura 1. Tipo de consumo de alcohol por sexo. $\mathrm{CN}=$ Consumo nulo; CsR = Consumo sin riesgo; $\mathrm{CR}=$ Consumo con riesgo; $\mathrm{CRD}=$ Consumo con riesgo de dependencia.

Finalmente se indaga si el rendimiento académico, evaluado a través de las variables del promedio actual y número de materias reprobadas de los participantes, está relacionado con el número de días a la semana que consumen alcohol, su puntaje obtenido en el AUDIT y basándose en este último, con el nivel de consumo de alcohol que presentan los estudiantes. Se realizó la prueba de Correlación de Pearson, y se encontró que el promedio académico de los participantes tiene una relación negativa, débil y significativa con el número de días a la semana que consumen alcohol $(R=-.23, p<$ $.001)$, con el puntaje del AUDIT $(R=-.25, p<.001)$ y con el nivel de consumo de alcohol $(R=-.23, p<.001)$. En cuanto al número de materias reprobadas, se encontró una relación positiva, débil y significativa con el número de días a la semana que hay consumo $(R=.25, p<.001)$, con el puntaje del AUDIT $(R=.19, p<.001)$, y con el nivel de consumo $(R=.19, p<.001)$. En la Tabla 2 se presentan los datos referentes a las correlaciones realizadas entre el rendimiento académico y las variables correspondientes al consumo de alcohol.

Tabla 2. Correlaciones obtenidas entre el rendimiento académico y el consumo de alcohol

\begin{tabular}{lccccc}
\hline & AUDIT & NCA & DSCA & PA & MR \\
\hline AUDIT & 1 & & & & \\
NCA & $.89^{* *}$ & 1 & & & \\
DSCA & $.60^{* *}$ & $.64^{* *}$ & 1 & & \\
PA & $-.25^{\star *}$ & $-.23^{\star *}$ & $-.23^{\star *}$ & 1 & \\
MR & $.19^{* *}$ & $.19^{* *}$ & $.25^{\star *}$ & $-.44^{\star *}$ & 1 \\
\hline
\end{tabular}

AUDIT = Puntaje obtenido en el AUDIT; NCA $=$ Nivel de Consumo de Alcohol; DSCA = Días a la semana que se consume alcohol; $\mathrm{PA}=$ Promedio Académico; $\mathrm{MR}=$ Materias reprobadas.

** Nivel de significancia correspondiente a $p<.001$

\section{Discusión}

El presente estudio tuvo como objetivos, describir el nivel de consumo de alcohol y asociar el nivel de consumo y el rendimiento académico en una muestra de estudiantes universitarios de la carrera de Psicología, en el estado de Hidalgo, México. Para esto, se realizó una evaluación en una muestra de estudiantes, utilizando una encuesta de datos sociodemográficos y el cuestionario AUDIT. Los resultados obtenidos en la evaluación del consumo de alcohol en la muestra de estudiantes universitarios, indican que $76 \%$ de los estudiantes han consumido alcohol al menos una vez en su vida; al hacer una distinción por sexo en este mismo rubro, se obtuvo $83.5 \%$ en los hombres y $73.2 \%$ en las mujeres; estos resultados son similares a los reportados en la ENCODAT 2016-2017 (4), en donde se reporta un porcentaje de $88.3 \%$ en los hombres y $67.3 \%$ en las mujeres para el mismo rubro. Situación similar se presentó para los alumnos que presentaron un puntaje igual o superior al correspondiente a "consumo de riesgo", el cual fue $18.2 \%$ en los participantes del estudio, mientras que en los resultados a nivel nacional fue de $19.3 \%$. Cabe destacar que el rango de edad para la encuesta nacional fue entre los 18 y 65 años, mientras que en el presente estudio la edad promedio fue de 19.8 años (DE = 2.13); esto podría sugerir que en los siguientes años, en las encuestas nacionales se reportará en un aumento en el nivel de consumo de alcohol en la población adulta.

Un dato relevante es el hecho de que el porcentaje de mujeres que consumen alcohol de manera excesiva ha ido en aumento en los últimos años; al hacer una diferencia por sexo en cuanto al nivel de consumo de alcohol en la muestra del estudio, se observa que los niveles de consumo son similares, concordando con los resultados obtenidos a nivel nacional. 4,5. Una explicación para este fenómeno son los cambios en el ámbito social y cultural del país, donde tradicionalmente se consideraba como negativo el hecho que las mujeres consumieran alcohol o tabaco; los cambios sociales relacionados con la búsqueda de la equidad entre hombres y mujeres, promueven que las mujeres se perciben con mayor libertad para consumir estas sustancias, sin que haya represión por parte de la sociedad, y por lo tanto, haya un aumento en la prevalencia de consumo de estas mismas sustancias. ${ }^{15,16}$

Por último, las correlaciones encontradas entre el puntaje del AUDIT, el nivel de consumo y el número de días a la semana que se consume alcohol, con el promedio académico y número de materias reprobadas, sugieren que al incrementarse el consumo de alcohol, el rendimiento académico tiende a disminuir, mientras que el número de materias reprobadas aumenta; esto concuerda 
con resultados previos donde se evidencia las consecuencias que produce el consumo excesivo de alcohol en el rendimiento académico de los estudiantes universitarios. ${ }^{6,7}$ También concuerda con lo descrito por la Organización Mundial de la Salud y la Asociación Americana de Psicología, las cuales mencionan que una de las principales consecuencias del consumo excesivo de cualquier sustancia adictiva es una disminución en el rendimiento del individuo en todas sus áreas. ${ }^{1,3}$

A pesar de que se hayan encontrado correlaciones significativas entre el rendimiento académico y el consumo de alcohol, existen diversas variables que también influyen para que el rendimiento académico de un estudiante universitario se vea disminuido; como el aumento en la exigencia académica, problemas familiares, la necesidad de algunos estudiantes de tener un trabajo de medio tiempo a la par de sus estudios, una mala elección de carrera por parte de la persona o falta de habilidades para solucionar problemas; sin embargo, algunas de estas variables también son consideradas como factores de riesgo para que un estudiante universitario consuma de manera excesiva alcohol. ${ }^{15,16}$

\section{Conclusiones}

En los resultados obtenidos, se observó una similitud en el nivel de consumo de alcohol de la muestra de estudiantes y los resultados obtenidos en las encuestas nacionales ENCODAT 2016-2017 y ENSANUT 2018. Los resultados obtenidos indican la necesidad de acciones, por parte de las instituciones de educación profesional, que tengan como objetivo la prevención y disminución del consumo de alcohol en los estudiantes; debido a la alta prevalencia de este problema, a los factores de riesgo presentes en este tipo de población y a las consecuencias que conlleva esta conducta en el propio rendimiento académico.

Esta investigación presenta ciertas limitantes a considerar; en primer lugar no se ocupó un muestreo probabilístico para obtener la muestra del estudio, por lo tanto, los resultados obtenidos no pueden ser generalizables a toda la población universitaria. Otra limitación es el hecho de que sólo se ocuparon cuestionarios estandarizados para evaluar el nivel de consumo de alcohol, dejando de lado otro tipo de instrumentos o técnicas que pudieran aportar datos más objetivos. Por último, la población estudiantil en donde se realizó la presente investigación tiene una proporción mayor de mujeres en comparación con la de hombres, esta diferencia puede influir en los resultados que se obtengan de la evaluación del consumo de alcohol.
Se sugiere para futuros estudios, que se haga uso de un muestreo probabilístico para obtener una muestra representativa y que los resultados que se obtengan puedan ser generalizables. Una segunda sugerencia es el uso de diversos instrumentos para evaluar el nivel de consumo de alcohol que sean válidos y confiables, así como evaluar otro tipo de variables que pudieran estar relacionadas como el nivel de ansiedad, estrés o síntomas de depresión en los estudiantes.

\section{Agradecimientos}

El presente estudio fue realizado gracias al recurso brindado por el Consejo Nacional de Ciencia y Tecnología (CONACyT) en formato de beca de manutención, así mismo, a la cooperación y apoyo brindado por las Psicólogas: Regina Navarro y Gala Fabela para la aplicación de los Cuestionarios en los participantes y la creación de la base de datos del estudio.

\section{Referencias}

[1] World Health Organization. Global status report on alcohol and health 2018. Geneva: WHO; 2018. Disponible en https://www.who.int/substance_abuse/publications/global_alcohol_re port/en/

[2] Comisión Interamericana para el Control del Abuso de Drogas, Organización de los Estados Americanos. Informe sobre el consumo de drogas en las Américas, 2019. Washington D. C.: OEA; 2019. Disponible

http://www.cicad.oas.org/main/pubs/Informe\%20sobre\%20el\%20con sumo\%20de\%20drogas\%20en\%20las\%20Am\%C3\%A9ricas\%20201 9.pdf

[3] American Psychiatric Association. Trastornos relacionados con sustancias y trastornos adictivos. En: Manual Diagnóstico y Estadístico de los Trastornos Mentales. 5ta. México: Editorial Médica Panamericana; 2014. p. 481-591

[4] Instituto Nacional de Psiquiatría Ramón de la Fuente Muñiz, Instituto Nacional de Salud Pública, Comisión Nacional Contra las Adicciones, Secretaría de Salud. Encuesta nacional de consumo de drogas, alcohol y tabaco 2016-2017: reporte de alcohol. 1.a ed. México: INPRFM; 2017. Disponible en https://www.gob.mx/salud\%7Cconadic/accionesy-programas/encuesta-nacional-de-consumo-de-drogas-alcohol-ytabaco-encodat-2016-2017-136758

[5] Instituto Nacional de Salud Pública, Instituto Nacional de Estadística y Geografía. Encuesta nacional de salud y nutrición 2018. México: INSP; 2018. Disponible en https://ensanut.insp.mx/encuestas/ensanut2018/doctos/informes/ensan ut_2018_presentacion_resultados.pdf

[6] Liguori G, Lonbaken B. Alcohol consumption and academic retention in first-year college students. Coll Stud J. 2015;49(1):69-77. Disponible https://www.ingentaconnect.com/content/prin/csj/2015/00000049/000 00001/art00008

[7] Davoren MP, Shiely F, Byrne M, Perry IJ. Hazardous alcohol consumption among university students in Ireland: a cross-sectional study. BMJ Open. 29 de enero de 2015;5(1):1-8. Disponible en https://www.ncbi.nlm.nih.gov/pmc/articles/PMC4759952/\#: :text=A $\% 20$ high $\% 20$ of $\% 2083.4 \% 20 \% 25 \% 20$ reporting,students $\% 20$ per $\% 20 \mathrm{w}$ eek\%20by\%202011. 
[8] Gutiérrez M, Lopera XM, Cruz LNS. Factores de riesgo y de protección asociados al consumo de alcohol en adolescentes. Psiconex. 2016;8(12):1-10. Disponible

https://www.scielo.br/scielo.php?script=sci_arttext\&pid=S0104$11692004000700010 \& \operatorname{lng}=$ en\&nrm=iso

[9] Bravo AJ, Pearson MR, Henson JM. Drinking to cope with depressive symptoms and ruminative thinking: a multiple mediation model among college students. Subst Use Misuse. 2016;52(1):52-62. Disponible en https://pubmed.ncbi.nlm.nih.gov/27668861/

[10] Bravo AJ, Prince MA, Pearson MR. College-related alcohol beliefs and problematic alcohol consumption: alcohol protective behavioral strategies as a mediator. Subst Use Misuse. 3 de julio de 2017;52(8):1$10 . \quad$ Disponible https://www.ncbi.nlm.nih.gov/pmc/articles/PMC5517017/

[11]Herman AM, Duka T. Facets of impulsivity and alcohol use: what role do emotions play? Neurosci Biobehav Rev. octubre de 2018;15. Disponible en https://pubmed.ncbi.nlm.nih.gov/30343823/

[12] Varlinskaya EI, Spear LP. Social consequences of ethanol: impact of age, stress, and prior history of ethanol exposure. Physiol Behav. septiembre de 2015;148:145-50. Disponible en https://pubmed.ncbi.nlm.nih.gov/25431835/

[13]Herrera-Chávez KJ, Linares-Rubio M, Díaz-Barajas D. Ambiente familiar e influencia social asociados al consumo de drogas ilegales y alcohol en adolescentes. Rev Educ Desarro. 2018;46:61-71. Disponible

http://www.cucs.udg.mx/revistas/edu_desarrollo/anteriores/46/46_Her rera.pdf

[14]Chung T, Creswell KG, Bachrach R, Clark DB, Martin CS. Adolescent binge drinking: developmental context and opportunities for prevention. Alcohol Res Curr Rev. 2017;39(1):5-15. Disponible en https://psycnet.apa.org/record/2019-05003-001

[15] García P, Trejo AG, Hinojosa L, Jiménez AA, Gracia GN. Prevalencia y creencias asociadas al consumo de alcohol en mujeres universitarias. Health Addict. 6 de agosto de 2020;20:81-7. Disponible en https://www.researchgate.net/profile/Pedro_Garcia_garcia/publication 1343494948_Prevalencia_y_creencias_asociadas_al_consumo_de_alc ohol_en_mujeres_universitarias/links/5f2da9eca6fdcccc43b2e404/Pre valencia-y-creencias-asociadas-al-consumo-de-alcohol-en-mujeresuniversitarias.pdf

[16]Alonso-Castillo MM, Un-Aragón LT, Armendáriz-García NA Navarro-Oliva EIP, López-Cisneros MA. Sentido de coherencia y consumo de alcohol en jóvenes universitarios. Investig Cienc. 2018;26(75):66-72. Disponible https://www.redalyc.org/jatsRepo/674/67457300008/html/index.html

[17] Guerrero-López CM, Muños-Hernández JA, Sáenz de Miera-Juárez B, Pérez-Núñez R, Reynales-Shigematsu LM. Impacto del consumo nocivo de alcohol en accidentes y enfermedades crónicas en México. Salud Pública México. 4 de marzo de 2013;55(2):282-8. Disponible en https://saludpublica.mx/index.php/spm/article/view/5126

[18]Dirección General de Atención al Ciudadano, Drogodependencias y Consumo, Consejería de Sanidad y Consumo. Guía de buenas prácticas y calidad en la prevención de drogodependencias en la comunidad autónoma de la región de Murcia: plan regional sobre drogas. España: Consejería de Sanidad y Consumo; 2010. Disponible en http://www.prevencionbasadaenlaevidencia.net/uploads/PDF/EG_Bue naspracticasprevencion_Murcia.pdf

[19]Secretaría General de la Organización de los Estados Americanos. Transferencia de las intervenciones breves para la prevención de recaídas en adicciones. Washington D. C.: OEA; 2017.

[20]Comisión Nacional Contra las Adicciones, Secretaría de Salud. Prevención del consumo de drogas. En: Informe Sobre la Situación del Consumo de Drogas en México y su Atención Integral. México: CONADIC; 2019. p. 35-43. Disponible en https://www.gob.mx/cms/uploads/attachment/file/477564/Informe_so bre_la_situacio_n_de_las_drogas_en_Me_xico_.pdf?utm_source=Dro $\mathrm{m} \% \mathrm{C} 3 \% \mathrm{~B} 3 \mathrm{manos}+\mathrm{Newsletter} \& u$ tm_campaign $=5810 \mathrm{baa} 060$ EMAIL_CAMPAIGN_2020_05_07_08_13_COPY_01\&utm_mediu $\mathrm{m}=$ email\&utm_term $=0 \_1754 \mathrm{eeb} 135-5810 \mathrm{baa} 060-53096605$

[21]Babor TF, Higgins-Biddle JC, Saunders JB, Monteiro MG. Cuestionario de identificación de los transtornos debidos al consumo de alcohol: pautas para su utilización en atención primaria. Geneva: OMS; 2001. Disponible en https://www.who.int/substance_abuse/activities/en/AUDITmanualSpa nish.pdf

[22] Medina-Mora E, Carreño S, De la Fuente JR. Experience with the alcohol use disorders identification test (AUDIT) in Mexico. En: Galanter M, editor. Recent Developments in Alcoholism, Vol 14: The Consequences of Alcoholism. New York: Plenum Press; 1998. p. 38396. [1] Dilla T, Valladares A, Lizán L, Sacristán JA. Adherencia y persistencia terapéutica: causas, consecuencias y estrategias de mejora. Aten. Primaria 2009; 41(6): 342-48. Disponible en https://link.springer.com/chapter/10.1007/0-306-47148-5_19 